\title{
Der Kaiser auf dem Forum. Das Forum Augustum als gebauter und geschriebener Raum öffentlicher Kommunikation
}

\begin{abstract}
Cibi uinique quocumque et tempore et loco appetentissimus, cognoscens quondam in Augusti foro ictusque nidore prandii, quod in proxima Martis aede Saliis apparabatur, deserto tribunali ascendit ad sacerdotes unaque decubuit.

Nach Speise und Wein hatte er zu jeder beliebigen Zeit und an jedem beliebigem Ort größtes Verlangen. Als er einmal auf dem Forum des Augustus eine gerichtliche Untersuchung anstellte und er vom Geruch einer Mahlzeit in die Nase wie vom Blitz getroffen wurde, die im nebenan gelegenen Tempel des Mars von den Saliern zubereitet wurde, verließ er den Richterstuhl, stieg hinauf zu den Priestern und legte sich zusammen mit ihnen zu Tisch.
\end{abstract}

So liest man in der Claudius-Vita des Sueton (Claud. 33,I = Lugli ı9o) - und man könnte (und kann mit Recht) meinen, die Begebenheit sei lediglich ein weiterer Beweis dafür, wie sehr Claudius selbst bei der Ausübung seiner kaiserlichen Amtsgeschäfte den elementaren Gelüsten unterworfen war. ${ }^{\mathrm{I}}$

Doch ist diese kurze Anekdote über das Biographische hinaus eine Art von Schlüsseltext für das als topographischen Rahmen genannte Augustusforum. ${ }^{2}$ Denn es zeigt sich in nuce die Polyfunktionalität dieses von Augustus geschaffenen, präzise geplanten städtischen Raumes sowie die Spannung zwischen der Umsetzung der Ziele des Bauherrn mit einem konsistenten ideologischen Konzept und der Praxis des täglichen Gebrauchs.

Die Claudius-Anekdote etwa ist ein Text, der nicht nur den Übergang vom Staatsmännischen zum Pedestren markiert, sondern auch die Beziehungen und Diskrepanzen zwischen den verschiedenen Ebenen der Nutzung des Forums deutlich werden lässt: der von Augustus herbeigeführten ideologischen Aufladung durch das Bau- und Bildprogramm und der davon repräsentativ gerahmten Funktion als Ort der Administration und Jurisdiktion und der Aneignung durch die Römer. Das Besondere an dem über Claudius berichteten Vorfall ist, dass die unterschiedlichen Verwendungsweisen simultan aufeinandertreffen (und damit in ihrer Disparität nicht zu übersehen sind), während das sonst üblicherweise durch zeitliche Staffelung kaschiert wurde (das Forum dient zu unterschied-

I Vgl. z.B. Kierdorf (I992) z. St.

2 Siehe die schon beinahe klassische Darstellung durch Zanker (i968); außerdem Spannagel (I999), zu den jüngsten Ausgrabungen Meneghini u. Valenzani (2007) 43-6o; generell Kienast (2009) 206f.; $242 \mathrm{f}$. 
lichen Zeiten des Tages und des Jahres unterschiedlichen, einander nicht tangierenden Zwecken). Es ist dies zugleich ein gewichtiger Spezialfall der bidirektionalen Kommunikation zwischen dem princeps und den Römern.

Im Folgenden sollen zunächst die antiken Texte ${ }^{3}$ durchgegangen werden, aus denen sich die verschiedenen Nutzungsformen des Augustusforums ablesen lassen - oftmals in literarischen Brechungen -, bevor auf dieser Basis ein genauerer Blick auf denjenigen Autor geworfen werden soll, der sich am ausführlichsten mit dem Augustusforum und dem Mars Ultor-Tempel auseinandergesetzt hat, auf Ovid (anhand der Fasti, dann auch der Ars amatoria und der Tristien). Es wird sich dabei zeigen, wie die Texte jeweils unterschiedliche Aspekte der Kommunikation zwischen Herrscher und Beherrschten hervortreten lassen.

Dass Claudius sich auf das von seinem Großonkel errichtete Forum begab, um richterlich tätig zu werden, entsprach zunächst der expliziten Zielsetzung des Bauherrn (Suet. Aug. 29 = Lugli 99):

Publica opera plurima exstruxit [sc. Augustus], e quibus vel praecipua: forum cum aede Martis Ultoris [...]. Fori exstruendi causa fuit hominum et iudiciorum multitudo, quae videbatur non sufficientibus duobus etiam tertio indigere; itaque festinatius necdum perfecta Martis aede publicatum est cautumque ut separatim in eo publica iudicia et sortitiones iudicum fierent.

Öffentliche Bauwerke errichtete er in großer Anzahl, von denen die wohl bedeutendsten sind: das Forum mit dem Tempel des Mars Ultor [...]. Grund für die Errichtung war die große Menge an Menschen und Gerichtsprozessen, die ein drittes Forum zu erfordern schien, da zwei nicht ausreichten. Deshalb wurde es übereilt, ohne dass noch der Marstempel vollendet gewesen wäre, der Öffentlichkeit übergeben, und es wurde dafür Sorge getragen, dass dort getrennt die öffentlichen Gerichtsprozesse und die Auslosung der Richter stattfinden sollten.

Augustus gab sich also viel Mühe damit, sein spektakuläres Projekt - spektakulär sowohl hinsichtlich der Dimensionen als auch der in jeder Beziehung prachtvollen Ausstattung nicht als Traditionsbruch erscheinen zu lassen, sondern als konsequente Fortsetzung der bisherigen Fora. Darin setzt sich die mit dem Konzept der res publica restituta verbundene Tendenz fort, die mit dem Prinzipat einhergehenden, auf eine Monarchie hindeutenden Neuerungen in republikanische Rhetorik einzukleiden und damit eine doppelte, je nach Kontext und Betrachter differenzierte Kommunikationsebene zu etablieren. Natürlich stand dem Betrachter zunächst die monumentale Inszenierung des julisch-claudischen Familienmythos (dazu unten genauer) vor Augen, aber die offizielle Zweckbestimmung ließ

3 Die Texte sind weitgehend zusammengestellt bei Lugli (I965) I5-37 (soweit vorhanden, ist die Quellennummerierung Luglis jeweils mit angegeben; bequem nutzbar, aber weniger umfassend ist van Heck [2002].). 
das als Rahmen und Begleiteffekt, nicht als Wesenskern erscheinen. Das macht sich zu Nutze, dass gerade die Gerichtsverhandlungen in Rom unter systematischen Gesichtspunkten als wesenseigen für Fora angesehen wurden, wie das mittelalterliche Exzerpt eines in augusteischer Zeit entstandenen Textes 4 belegt (Paul. Fest. p. 74,I5f.):

Forum sex modis intellegitur; primo [...], alio, in quo iudicia fieri, cum populo agi, contiones haberi solent.

,Forum‘ kann auf sechs Weisen verstanden werden: erstens [...], zweitens [als ein Platz,] auf dem Gerichtsverhandlungen stattzufinden, mit dem Volk verhandelt zu werden und Volksversammlungen abgehalten zu werden pflegen.

Diese spezifische Verwendung des Augustusforums für die Jurisdiktion blieb auch über das Ende der julisch-claudischen Dynastie hinaus mindestens bis in die Zeit Traians bestehen, unabhängig davon, dass mit dem Traiansforum nun ein noch größerer Raum für solche Tätigkeiten verfügbar war. 5

Claudius war also ausgegangen, um einer seiner genuinen herrscherlichen Pflichten nachzukommen, nämlich um eine Gerichtsverhandlung abzuhalten. Nun war das Areal des Forums weitläufig und durch die Säulengänge und Nischen auf den ersten Blick v.a. für Fremde nicht leicht überschaubar. Es erwies sich deshalb als notwendig, die Prozessankündigungen um genauere Ortsangaben zu ergänzen, um es den Parteien und den möglichen Zuschauern zu erleichtern, die jeweilige Verhandlung tatsächlich zu finden und kein Versäumnisurteil zu riskieren. Das belegen Ankündigungen, wie sie in den Tabulae Herculanenses und Tabulae Pompeianae Sulpiciorum 6 erhalten sind und die den Ort der Gerichtsverhandlungen Romae in foro Augusto jeweils näher erläutern:7
Ante statuam Cn. Senti Saturnini / triumphalem hora tertia ${ }^{8}$
ante statuam Gracci / ad columnam quartam prox / ume gradus 9
ante aedes Martis Ultoris hora tertia ${ }^{\text {IO }}$
ante / aram Martis Ultoris proxume / gradus hora quarta ${ }^{\mathrm{II}}$

\footnotetext{
4 Lhommé (2OII).

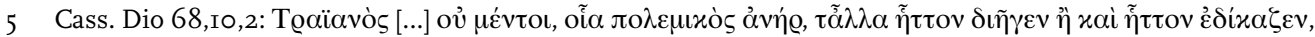

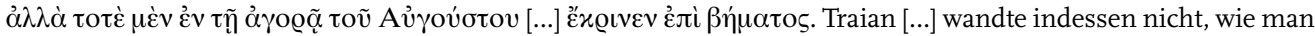
von einem Kriegsmann hätte erwarten sollen, den anderen Aufgaben geringere Aufmerksamkeit zu oder beschäftigte sich weniger mit Rechtsprechung: ganz im Gegenteil, er war bald auf dem Augustusforum [...] auf einem Tribunal als Richter tätig.

6 Camodeca (I999).

7 Siehe Carnabuci (I996).

8 Camodeca (I999) Nr. 27; Wolf (2010) Nr. I4; Carnabuci (I996) 77-85.

9 Camodeca (I999) Nr. I9; Wolf (2010) Nr. ı9; Carnabuci (ı996) 88-90.

Io Carnabuci (I996) $56 \mathrm{f}$.

II Camodeca (I999) Nr. I5; Wolf (2010) Nr. I5; Carnabuci (I996) 86-88.
} 
Die Rechtsgelehrten, die sich das Augustusforum zum Standort ausgewählt hatten, waren nicht immer auf Augenhöhe mit dem erhabenen Ambiente, wie sich aus einem Seitenhieb Martials gegen einen Plagiator schlussfolgern lässt (Mart. 7,5I = Lugli I86): ${ }^{\text {I2 }}$

\author{
Mercari nostras si te piget, Urbice, nugas \\ Et lasciva tamen carmina nosse libet, \\ Pompeium quaeres - et nosti forsitan - Auctum; \\ Ultoris prima Martis in aede sedet: \\ iure madens varioque togae limatus in usu \\ non lector meus hic, Urbice, sed liber est.
}

Wenn du unsere Kleinigkeit nicht mehr verkaufen willst, Urbicus, und du dennoch frivole Gedichte kennenlernen willst, dann suche den Pompeius - und du kennst ihn vielleicht den Pompeius Auctus, er sitzt ganz vorne im Tempel des Mars Ultor, triefend von Rechtsgelehrtheit und mit ausgefeilter wechselnder Togamode. Hier ist nicht mein Leser, Urbicus, sondern mein Buch.

Auch in diesen Versen finden wir eine das Augustusforum differenzierende lokale Angabe, die den Aufenthaltsort des Plagiators und Rechtsgelehrten (von zweifelhafter Reputation) Pompeius Auctus beschreibt. Dabei bildet die monumentale Kulisse den gewollten Kontrast zur Erscheinung des Winkeladvokaten mit dem aufschlussreich sprechenden Namen. Solche Ortsangaben bilden auch den lokalen Rahmen von Claudius' nicht vorhersehbarem Rollenwechsel, in dem er seine auch durch das Tribunal optisch herausgehobene Stellung als Richter mit derjenigen des pontifex maximus vertauschte, sich allerdings sowohl tatsächlich als auch übertragen auf dieselbe Ebene mit der betreffenden Gruppe begab. Es kann ohne weiteres sein, dass er seinen Richterstuhl in der Nähe des Mars Ultor-Tempels hatte den Angaben aus den beiden Tabulae sowie bei Martial entsprechend -, just wo auch die Salier ihr Amtslokal hatten.

Noch in der Spätantike sind die mansiones Saliorum Palatinorum auf dem Augustusforum inschriftlich belegt (CIL VI, 2158). Der Grund dafür, dass sie hier eine Dependance zu ihrem eigentlichen Amtslokal auf dem Palatin hatten, dürfte in ihrer Zugehörigkeit zum Marskult liegen, ${ }^{13}$ dessen wichtigster Tempel sich nun seit Augustus eben auf dessen Forum befand. Da die Salier nicht nur den Marskult ausübten, sondern auch spektakuläre Gastmähler abhielten (vgl. Cic. Att. 5,4,I epulari Saliarem in modum), kam es zu dieser ganz speziellen Interaktion zwischen Kaiser und Priestern, die allerdings selbst für Claudius eine Ausnahme dargestellt haben dürfte.

Die Salier waren nicht die einzige Priesterschaft auf dem Augustusforum. Auch das ebenfalls den römischen Eliten zugehörige Kollegium der Arvalen machte den Ort zum

I2 Schöffel (2002) z. St.

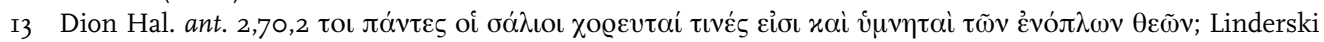
(200I); Edelmann (2003) 203. 
Schauplatz seiner Opferhandlungen:I4 item in foro Aug(usto) Marti Ultori taurum - vielleicht auch, weil Mars im Carmen Arvale an prominenter Position mit satur fu fere Mars ${ }^{15}$ angerufen wurde. Das Augustusforum geriet also zum Schauplatz bereits etablierter Kulte, was seine Sonderstellung weniger provokativ erscheinen ließ.

$*$

Die bei Martial und in den Tabulae erwähnten Statuen waren ursprünglich natürlich nicht als Merkhilfen gedacht, um die Plätze zu finden, wo sich die Juristen verdingten. Vielmehr hatte sich Augustus persönlich um sie gekümmert und sie zu einem wesentlichen Teil der politischen Botschaft und Träger der optischen Organisation der Anlage werden lassen: ${ }^{16}$ Sie flankierten den Blick auf das zentrale Bauwerk, auf den Mars Ultor-Tempel, indem sie in den beiden Portiken aufgereiht standen: ${ }^{17}$

Itaque et opera cuiusque manentibus titulis restituit et statuas omnium triumphali effigie in utraque fori sui porticu dedicavit, professus et edicto: commentum id se, ut ad illorum vitam velut ad exemplar et ipse, dum viveret, et insequentium aetatium principes exigerentur a civibus. (Suet. Aug. 3I)

Also richtete er auch Werke mit beständigen Inschriften wieder her und weihte Statuen aller mit triumphalem Aussehen in beiden Portiken seines Forums, was er in einem Edikt kund tat. Er habe das getan, damit nach jener Leben wie nach einem Vorbild er selbst, solange er leben würde, und die Principes der folgenden Zeitalter von ihren Mitbürgern zu handeln aufgefordert würden.

Das war Augustus so wichtig, dass er sich zumindest in einigen Fällen persönlich um die Inschrift kümmerte, mit der diese exemplarischen Männer gewürdigt wurden (Plin. nat. 22,6,I3 = Lugli II7):

Quod et statuae eius [scil. Scipionis Aemiliani] in foro suo Divus Augustus inscripsit.

Diese Inschrift setzte auf die Statue des Scipio Aemilianus auf seinem Forum der Göttliche Augustus.

Leider haben wir den genauen Wortlaut dieser Inschrift nicht durch einen einschlägigen Fund bestätigt, ${ }^{18}$ aber es ist doch eine erkleckliche Anzahl von (teils fragmentierten) tituli

I4 z.B. Scheid (I998) 70, Nr. 2

I5 Vgl. Norden (I939) I36-I4I; Edelmann (2003).

I6 Zanker (I968), jetzt umfassend Geiger (2008).

I7 Ausführlich dazu Geiger (2008) II7-I62.

I8 Geiger (2008) I4I. 
erhalten. ${ }^{19}$ Sie konnten so kurz und bündig sein wie diejenige, die jeden rationalistischen Zweifel an der göttlichen Natur des Stadtgründers beiseite wischte: ${ }^{20}$

$\operatorname{Ro}[$ mulo, $] / M[$ artis f(ilio, ) ] / Ur[bis conditori $]$.

Für Romulus, den Sohn des Mars, den Gründer der Stadt

Die folgende Inschrift gibt auf der anderen Seite einen Eindruck vom möglichen Umfang dieser Inschriften, die durchaus narrative Elemente enthalten konnten. ${ }^{2 \text { I }}$ Obendrein ist mit dem erwähnten Ti. Gracchus sogar eine mögliche Verbindung zum in den Tabulae genannten Gracchus ${ }^{22}$ hergestellt:

Q(uintus) Caec[ilius Q(uinti) f(ilius) Metellus]

[Numidicus]

[cens(or) co(n)s(ul) pr(aetor) aug(ur)] //

[-----]

[--- complures civitates in]

[pote]state[ $m$ accepit de rege Iugurtha triumphavit]

[ce]nsor L(ucium) Eq[uitium qui se Ti(beri) Gracchi filium mentiebatur]

[in cen]s[um non recepit ---]

$[---] S[---]$

$[----?]$

Quintus Caecilius Metellus, der Sohn des Quintus, Numidicius, Censor, Konsul, Praetor, Augur, empfing eine Reihe von Staaten in seine Herrschaft, triumphierte über den König Iugurtha und nahm als Censor den Lucius Equitius, der sich lügnerisch als Sohn des Tiberius Gracchus ausgab, nicht in den Census auf.

Am besten geeignet für einen Treffpunkt waren aber gewiss Statuen, die eine auch optisch wahrnehmbare, rasch identifizierbare Besonderheit aufwiesen (Gell. 9,II,IO = Lugli I29):23

Statuam Corvino isti divus Augustus in foro suo statuendam curavit. In eius statuae capite corvi simulacrum est rei pugnaeque, quam diximus, monimentum.

Eine Statue für diesen Corvinus ließ der göttliche Augustus auf seinem Forum errichten. Auf dem Haupt der Statue ist das Bild eines Raben als Andenken an die Sache und die Schlacht, von der wir gesprochen haben.

I9 Degrassi (I937).

20 Der Standort der Romulus-Statue und der gegenüberliegenden Aeneas-Statue in den beiden den Tempel flankierenden Hemizyklien ist auch nach den Ausgrabungen der letzten Jahre unbezweifelt. Unklar ist, ob die beiden neu zu Tage gekommenen Hemizyklien am Nordwestende eine ähnliche konzeptionelle Bedeutung hatten; kurz zum aktuellen Stand Geiger (2008) I08-IIO mit Abb. 5, zum Text der Inschrift ibd. I37.

2I Geiger (2008) I53f.

22 Geiger (2008) I23-I26; I5I.

23 Geiger (2008) 34 stellt einen Bezug zu den exempla aus republikanischer Zeit her. 
Joseph Geiger hat in seiner Monographie zur Statuengalerie dieses Forums auch die Frage nach der kommunikativen Absicht des Augustus aufgeworfen: Ob nicht diese Verbindung von Bild und Text darauf abzielte, eine möglichst breite Schicht (und nicht nur die Bildungseliten) in das damit verbundene Programm einzubeziehen. ${ }^{24}$ Diese Mischung aus plakativen Botschaften und feinsinnigen Anspielungen etwa durch architektonische Zitate macht das Forum Augustum zum Musterbeispiel öffentlicher Kommunikation im frühen Prinzipat.

Augustus kümmerte sich sogar, wie Gellius in der soeben zitierten Passage berichtet, ${ }^{25}$ um die ikonographischen Details, offenbar aus Sorge darüber, nur ja nicht die mit der Statuengalerie verbundene Botschaft, die Systematisierung der memoria und die auf die $\mathrm{Zu}-$ kunft weisende Ausrichtung aus der Hand zu geben. Gerade die Verbindung von iulischer Familiengenealogie und allgemein-römischer, in personale Konzepte gegossener Geschichte - womit auch eine Kanonisierung dieser Geschichte und der sie tragenden Männer verbunden war $-{ }^{26}$ war erst unter den Bedingungen des Principats möglich und damit ein besonderes Fingerspitzengefühl erforderndes Novum.

Es ist nicht nötig, hier Bekanntes und Allzubekanntes über die Architektur und Bestimmung des Augustusforums zu wiederholen. ${ }^{27}$ Natürlich hatte Augustus schon von Anfang an keinen Zweifel daran gelassen, dass er die Deutungshoheit über seine Gabe an das römische Volk behalten wollte (Mon. Anc. 2I,I = Lugli 9I): 28

In privato solo Martis Ultoris templum [f]orumque Augustum [ex mani]/biis feci.

Auf privatem Grund und Boden errichtete ich den Tempel für Mars Ultor und das Augustusforum aus Kriegsbeute.

Er war der Bauherr, der auch die damit verbundenen Inhalte bestimmte. Daran änderte auch nichts, dass Augustus auf auftrumpfende Enteignungen verzichtete, sondern auf gutnachbarschaftliche Beziehungen setzte. ${ }^{29}$ Möglicherweise stand der Bau auch durch solche Rücksichtnahmen unter keinem ganz glücklichen Stern, wie der bei Macrobius bewahrte Seufzer des Augustus illustriert. $3^{\circ}$

24 Geiger (2008) 69-73.

25 Vgl. Stevenson (2004), bes. I38. Generell zu Gellius jetzt Keulen (2009).

26 Geiger (2008) 86.

27 Vgl. etwa auf aktuellem archäologischem Stand Ungaro (2007) 99-169.

28 Zur Bedeutung der Angabe ex manibiis (singulär für die Bauten des Augustus, aber auch schon für das Forum Iulium belegt) vgl. Bastien (2007), bes. 33I-337 über die republikanischen Vorläufer dieser Praxis (dreiundvierzig solche Weihungen ex manu[i]biis sind dort vor Augustus aufgelistet). Siehe auch zuletzt Scheid (2007) mit dem Kommentar ad loc.

29 Suet. Aug. 56 = Lugli Ioo: Forum angustius fecit non ausus extorquere possessoribus proximas domos (Das Forum ließ er schmaler errichten, da er es nicht wagte, den Besitzern die angrenzenden Häuser zu entwinden.). Die daraus resultierende Abschrägung an den Seiten neben dem Mars Ultor-Tempel ist auch in der Forma Urbis bewahrt: Meneghini u. Valenzani (2007) 56, fig. 48.

30 Macrob. Sat. 2,4,9 = Lugli I03: Cum multi Severo Cassio accusante absolverentur, et architectus fori Augusti expectationem operis diu traheret, ita iocatus est „Vellem Cassius et meum forum accuset“. (Als viele, die Severus Cassius anklagte, frei gesprochen wurden und der Architekt des Augustusforums die Erwartung auf die Vollendung 
Das Resultat für das Projekt des Augustus war allerdings so beeindruckend, dass Plinius nicht zögerte, es mit den etablierten Weltwundern gleichzusetzen (Plin. nat. 36,102 = Lugli 97):31

Non inter magnifica basilicam Pauli columnis e Phrygibus mirabilem forumque divi Augusti et templum Pacis Vespasiani Imp. Aug., pulcherrima operum, quae umquam vidit orbis?

Und sind nicht unter den staunenswerten Dingen die Basilica des Paulus, die wegen ihrer Säulen aus phrygischem Marmor wunderbar ist, und das Forum des vergöttlichten Augustus und der Friedenstempel des Kaisers Vespasian, die schönsten der Bauwerke, die jemals der Erdkreis gesehen hat?

Charakteristisch für die Konstruktion des Prinzipats in seiner Frühzeit ist, wie Augustus konsequent die private Motivation und die Sorge um die res publica als ganze zusammenführte: Ausgangspunkt für das gesamte Projekt war die Rache an den Caesarmördern gewesen (Suet. Aug. 29,2 = Lugli 99):

Aedem Martis bello Philippensi pro ultione paterna suscepto voverat.

Den Tempel des Mars hatte er im für die Rache für seinen Vater begonnen Krieg von Philippi gelobt.

Doch mit hoher Wahrscheinlichkeit hatte der künftige Augustus zu diesem Zeitpunkt in den 4 oer Jahren weder eine genaue Vorstellung über die architektonische Einbindung des Tempels in ein erst zu schaffendes Forum noch über den genauen Standort. ${ }^{2}$ Erst die zum Sieg überhöhte Rückgewinnung der von Crassus an die Parther verlorenen Feldzeichen, die ebenfalls dem Mars Ultor zugeschrieben wurde, ließ das Projekt konkret werden. Damit wurden die alltagspraktischen Effekte, nämlich dass der nach der Justizreform des Augustus spürbar angestiegene Bedarf an Gerichtsplätzen befriedigt werden konnte, durch die monumentale Repräsentation des ideologischen Gehalts zwar überstrahlt, aber nicht verdrängt. Diese Verbindung aus Eigenem und Allgemeinem zeigt sich - wie erwähnt - in der Konzeption der Statuengalerie, die den iulischen Familienmythos mit den großen Männern der römischen Geschichte konfrontierte. Es setzte sich am Tempel des Mars Ultor fort, wo sowohl Mars als auch Venus bildlich repräsentiert waren, nicht nur als archetypisches Sinnbild von Krieg und Frieden, Eros und Thanatos, sondern auch als Stammvater Roms und Stammmutter der gens Iulia. ${ }^{33}$

des Werks immer länger ausdehnte, scherzte er: „Ich wollte, Cassius würde auch mein Forum anklagen.“). Dass diese Bauzeit mit der legendären Bauzeit der römischen U-Bahn im 20. und 2I. Jahrhundert auffällig übereinstimmt, sei als Elementarparallele angemerkt.

3I Vgl. Carey (2003) I49-I5I.

32 Siehe zuletzt Geiger (2008) 53-60.

33 Vgl. Zanker (I968) I4f.; Galinsky (I996) 2I2 über die Verbindung zum pietas-Konzept der Aeneis. 
Angesichts dieser Bedeutung überrascht es, dass wir - anders als für Claudius - kein einziges explizites Zeugnis für die Anwesenheit des Augustus auf seinem Forum haben.34 Doch es ist zuversichtlich zu vermuten, dass er sich auch auf dem Forum zeigte, als er dort (ausnahmsweise, da eine Tiberüberschwemmung dazu zwang) die ludi Martiales abhalten ließ.35

Aber aus einigen, bisweilen etwas bizarr anmutenden Zeugnissen wissen wir, dass er jedenfalls in effigie auf seinem Forum zugegen war. Auf Betreiben Sejans unterstellte Tiberius der Agrippina, der Witwe des Germanicus, sie wolle zu einer dramatischen öffentlichen Handlung greifen, um ihre Bedrohung offenbar werden zu lassen, und sich dafür das Standbild des Augustus zu Nutze machen (Tac. ann. 4,67,4):36

[...] ultroque struebantur, qui monerent perfugere ad Germaniae exercitus vel celeberrimo fori effigiem divi Augusti amplecti populumque ac senatum auxilio vocare.

[...], ja, man stellte Leute ab, die ihnen Winke geben mussten, sich zu den germanischen Heeren zu flüchten, oder, wenn das Forum am besuchtesten wäre, das Bild des verewigten Augustus zu umfassen und das Volk und den Senat um Hilfe anzurufen.

Das scheint keine Erfindung des Tacitus zu sein, denn auch Sueton weiß von dem Vorfall (Suet. Tib. 53,2 = Lugli I04):

Novissime calumniatus modo ad statuam Augusti modo ad exercitus confugere velle, Pandatariam relegavit.

Schließlich beschuldigte er sie, dass sie zu Füßen der Augustusstatue oder auch beim Heer Zuflucht nehmen wollte und verbannte sie nach Pandataria.

34 Dass Kaiser Spaziergänge durch die Stadt unternahmen, belegt mutatis mutandis wiederum für Claudius, der Bericht bei Plin. epist. I,I3,3: At hercule memoria parentum Claudium Caesarem ferunt, cum in palatio spatiaretur audissetque clamorem, causam requisisse, cumque dictum esset recitare Nonianum, subitum recitanti inopinatumque venisse. - Wenn der Palatin, wie es die jüngsten Untersuchungen nahe legen, schon zur Zeit Claudius in toto das Areal des Herrschersitzes bildete, dann wäre Claudius innerhalb dieser Palastanlage unterwegs gewesen (vgl. die zusammenfassende Darstellung von Cecamore [2002], bes. 213-219), was aber nichts am Flanieren in einem herrscherlichen architektonischen Ensemble ändert.

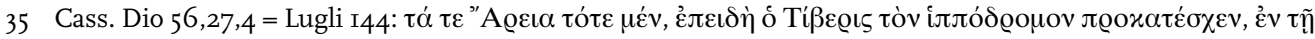

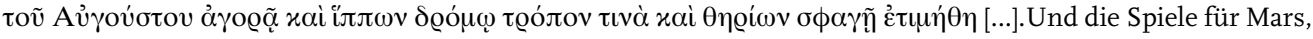
nachdem der Tiber den Circus überflutet hat, wurden auf dem Augustusforum abgehalten und wurden mit einem Pferderennen und einer Tierhatz begangen. - Vgl. Galinsky (I996) I99: „The forum was meant to be more than a museum.“

36 Nichts dazu inhaltlich bei Koestermann (I963) ad loc.; vgl. zum Asylmotiv (Zuflucht zu einer Herrscherstatue) 3,36,I. 
Diese Statue muss von beeindruckender Größe gewesen sein (und ich möchte pace Spannagel37 gerne bei der Deutung auf Augustus bleiben), denn es gibt keinen vernünftigen Grund, in diesem Punkt der Aussage Martials zu misstrauen (Mart. 8,44,3; 6-9 = Lugli 9): 38

At tu, miser Titulle, nec senex vivis
$[\ldots .$.
foroque triplici sparsus ante equos omnis
aedemque Martis et colosson Augusti
curris per omnes tertiasque quintasque.

Aber du, armer Titullus, genießt nicht dein Leben im Alter. Du ziehst über die drei Fora vor den Rittern und den Tempel des Mars und den Koloss des Augustus stets in der Zeit zwischen der dritten und fünften Stunde.

Das Augustusforum war auch Schauplatz eines ex officio vollzogenen Akts des Vandalismus39 (Plin. nat. 35,93 = Lugli II5):

Romae Castorem et Pollucem cum Victoria et Alexandro Magno, item Belli imaginem restrictis ad terga manibus, Alexandro in curru triumphante. Quas utrasque tabulas divus Augustus in fori sui celeberrimis partibus dicaverat simplicitate moderata; divus Claudius pluris existimavit utrisque excisa Alexandri facie divi Augusti imagines addere.

In Rom gibt es das Bild des Castor und Pollux mit der Victoria und Alexander dem Großen, ebenso ein Bild des Krieges mit auf den Rücken gebundenen Händen und Alexander im Triumphwagen. Beide Bilder hatte der göttliche Augustus auf den meist besuchten Teilen seines Forums mit großer Mäßigung und Einfachheit aufgestellt. Der göttliche Claudius hielt es für mehr wert, beiden Bildern das Gesicht des Alexander herausschneiden zu lassen und die Bilder des göttlichen Augustus hinzuzufügen.

37 Spannagel (1999) 303-306, so auch Geiger (2008) 98.

38 Carnabuci (1996) 36-43. Schöffel (2002) 378-39I (384,4: gemeint sei die Kolossalstaue des Domitian).

$39 \mathrm{Zu}$ hübsch (wenn auch aus archäologischen Gründen eher unwahrscheinlich) wäre es natürlich, wenn der unter Tiberius vorgekommene Fall von Vandalismus mit dem Augustusforum in Verbindung gebracht werden könnte, wie Lugli in seiner Quellensammlung vermutet (Suet. Tib. 58, I = Lugli I05):

Statuae quidam Augusti caput dempserat, ut alterius imponeret; acta res in senatu et, quia ambigebatur, per tormenta quaesita est. damnato reo paulatim genus calumniae eo processit, ut haec quoque capitalia essent: circa Augusti simulacrum seruum cecidisse, uestimenta mutasse, nummo uel anulo effigiem impressam latrinae aut lupanari intulisse, dictum ullum factumue eius existimatione aliqua laesisse.

Jemand hatte einer Augustusstatue den Kopf abgenommen, um einen anderen aufsetzen zu lasen. Die Angelegenheit kam vor den Senat, und da die Beweise nicht zwingend waren, schritt man zur Folter. Der Angeklagte wurde verurteilt, und allmählich ging man in dieser Art von Anklage so weit, dass als todeswürdiges Verbrechen angesehen wurde, wenn jemand in der Nähe eines Augustus-Bildes einen Sklaven auspeitschen ließ oder seine Kleider wechselte, ein Geldstück oder einen Ring mit dem Bild des Augustus auf den Abtritt oder in ein Bordell mitnahm, oder einen Ausspruch oder eine Maßnahme des Augustus zu kritisieren wagte. 
Claudius vereindeutigt damit, was Augustus durch die Auswahl seiner Bilder nur angedeutet hatte: ${ }^{\circ}$ die Überwindung des Krieges als Voraussetzung für die pax Augusta ${ }^{\mathrm{I}}$ und die imitatio Alexandri,42 die die Sieghaftigkeit dieses Friedens zum Ausdruck brachte. Wie lange sich diese Entdisambiguierung tatsächlich halten konnte ist nicht klar, der überwundene Krieg war aber wohl bis in die Spätantike auf dem Augustusforum zu sehen, wie eine Erläuterung bei Servius nahelegt. 43

Schon bevor Claudius den Augustus in der gerade genannten Weise bildhaft auf seinem Forum hatte ankommen lassen, war auch der römische Senat tätig geworden (Mon. Anc. 35 = Lugli Io9):44

Tertium decimum consulatum cum gerebam, senatus et equester ordo populusque Romanus universus appellavit me patrem patriae, idque in vestibulo aedium mearum inscribendum et in curia Iulia et in foro Aug. sub quadrigis quae mihi ex s.c. positae sunt censuit.

Als ich mein dreizehntes Konsulat ausübte, da nannte mich der Senat, der Ritterstand und das römische Volk einmütig Vater des Vaterlandes und sie sorgten dafür, dass das im Vestibulum meiner Häuser inschriftlich festgehalten wurde, sowie in der Curia Iulia und auf dem Augustusforum unter der Quadriga, die für mich aufgrund eines Senatsbeschlusse dort errichtet worden war.

Das bisher erarbeitete Spektrum herrscherlicher Präsenz reicht also vom Kulinarischen bis zur symbolischen Repräsentation. Dieser polyvalenten Bespielung entspricht die Vielstimmigkeit der Rezeption. Es ist stets nach den Kontexten zu fragen, auch nach den Tages-, Monats- und Jahreszeiten. An den Vormittagen wird es dort ganz anders ausgesehen haben als am Nachmittag, an den dies fasti anders als an den dies nefasti, wenn die dichte Menge der Rechtsuchenden fehlte. Die ausländischen Delegationen dürften sich kaum durch die Volksmenge gedrängt haben. Und wenn gar ein Triumphator kam, um die Tri-

40 Zanker (1968) 23f. nimmt an, dass das geschehen sei, weil die ursprünglich mit der Aufstellung verbundene Absicht nicht mehr erkennbar gewesen sei.

4I Schmitzer (2004).

42 Prinzipiell zuletzt Tisé (2002) (v.a. zur Zeit der mittleren Republik) und Kühnen (2008).

43 Serv. Aen. I,29I = Lugli II6: „Furor impius intus“ ut superius diximus propter bella civilia, quae gesta sunt contra Brutum et Cassium ab Augusto in Philippis, contra Sextum Pompeium ab Augusto in Sicilia. aut sicut quidam tradunt "furor impius intus" non in aede Iani, sed in alia in foro Augusti introeuntibus ad sinistram, fuit bellum pictum et furor sedens super arma devinctus eo habitu quo poeta dixit. - FUROR IMPIUS INTUS, wie wir vorhin sagten wegen der Bürgerkriege, die gegen Brutus und Cassius von Augustus bei Philippi geführt wurden und gegen Sextus Pompeius von Augustus in Sizilien. Oder wie andere überliefern, bezieht sich FUROR IMPIUS INTUS nicht auf den Tempel des Ianus sondern auf einen anderen, der für diejenigen, die das Augustusforum betreten, auf der linkens Seite liegt: Es war dort ein gemalter Krieg und ein Furor, der gefesselt über Waffen saß, in der Gestalt, wie es der Dichter sagte.

44 Scheid (2007), ad loc. mit Hinweis auf Rich (1998), bes. II5-I25. Ob auf der Quadriga nun eine Augustusstatue war oder nicht (so Rich [I998]), entscheidend ist die unverkennbare symbolische Präsenz des Herrschers als Sieger auf seinem Forum. 
umphinsignien im Mars Ultor-Tempel zu deponieren, dann war das trotz der imposanten Kulisse ein fast intimes Seitenstück zum eigentlichen Triumphzug. Denn all die Wagen, Beutestücke, Soldaten und Gefangenen passten schlicht nicht durch den schmalen Zugang. 45

Überhaupt scheint Augustus die Wahl der Zugänge sehr genau kalkuliert zu haben. Denn wer das Augustusforum betreten wollte, sah keineswegs schon von ferne, was für ein Anblick sich ihm bieten würde. ${ }^{6}$ Es gab keine weitreichenden Blickachsen auf den Mars Ultor-Tempel hin, vielmehr war der Erstbesucher schlagartig überwältigt, wenn sich ihm die Marmorpracht öffnete. 47 Er befand sich in einem klar definierten, neuartigen Raum. Und dieser Laborcharakter macht das Augustusforum für eine exemplarische Untersuchung von Kommunikationsräumen so geeignet.

$$
*
$$

Welch tiefen Eindruck diese Rauminszenierung auf die Zeitgenossen machte, können wir durch einen glücklichen literarischen Zufall nachvollziehen. Denn Ovid schildet im 5. Buch der Fasti, das nur kurz nach der Einweihung des Forums entstanden ist, $4^{8}$ einen Besuch dort. Doch bevor der elegische Erzähler dazu kommt, sich selbst ein Bild zu verschaffen, wird er von der Ankunft eines weiteren Besuchers überrascht (Ov. fast. 5,549f. = Lugli I68):49

Fallor, an arma sonant? non fallimur, arma sonabant:

Mars venit et veniens bellica signa dedit.

Täusche ich mich? Oder klirren (da) Waffen? Ich täusche mich nicht, es klirrten (da) Waffen! Mars kommt, und im Kommen gab er Kriegssignale.

Die Epiphanie des Gottes Mars kündigt sich mit kriegerischen Tönen an. Mars ist sich also vor der Autopsie noch nicht ganz sicher, was ihn da erwartet. Denn - so Alessandro Barchiesi -50 die Verbindung von arma und sonare weist zunächst auf einen aggressiven, krie-

45 Über mögliche despektierliche Äußerungen Juvenals (sat. I,95f.; I29-I3I) gerade über diese Funktion des Forums vgl. Larmour (2007) I8I-I85.

46 Galinsky (I996) $202 \mathrm{f}$.

47 Zanker (I968) 6f.: „Es ist bezeichnend, dass sowohl das Caesar- wie das Augustusforum als Tempelplätze konzipiert sind. Diese waren ihrer Bestimmung gemäß seit je abgeschlossene Bezirke. [...] Auch auf das republikanische Forum Romanum mündete eine Vielzahl von Straßen und Wegen. [...] Das Augustusforum war die erste konsequent als Repräsentationszentrum konzipierte Platzanlage. Es sollte weder von Marktbetrieb noch von Verkehr belastet sein, es war nicht für Volksversammlungen bestimmt, sondern sollte [...] dem täglichen Verwaltungs- und Schulbetrieb, in erster Linie aber den Gerichten dienen."

48 Vgl. Herbert-Brown (I994) 95-I08; wenig ergiebig Riedl (I989); vgl. Schmitzer (2003) 210-2I4.

49 Übersetzung hier und im folgenden modifiziert nach Bömer (I957/58).

50 Barchiesi (2002). 
gerischen Kontext hin. ${ }^{\text {I }}$ Auch im Pentameter mit dem tatsächlichen Erscheinen des Gottes ist der kriegerische Duktus weiter aufrecht erhalten..$^{2}$

Nur vorsichtig nähert sich Mars dem ihm gewidmeten Baukomplex - dem ersten Marstempel innerhalb des pomerium - und sein Erstaunen setzt Ovid in eine der ungewöhnlichsten Ekphraseis in den Fasti um,53 nämlich aus der Perspektive eines Gottes (Ov. fast. 5,55If. = Lugli I68):

Ultor ad ipse suos caelo descendit honores templaque in Augusto conspicienda foro.

Der Rächer steigt selbst vom Himmel herab, um seine Ehren und seinen Tempel auf dem Forum des Augustus anzuschauen.

Durch das augusteische Epitheton Ultor 54 wird im Nachhinein auch das kriegerisch-aggressive Erscheinungsbild des Mars plausibel, nämlich als ekphrastische Umsetzung der Statue in seinem Tempel55 auf dem hier erstmals so genannten Augustusforum..$^{6}$ Auf die unter antiquarischem Gesichtspunkt schon lange geführte Forschungsdiskussion, ob Ovid das Weihedatum des Tempels oder ein anderes Fest behandelt, soll hier nicht eingegangen werden, 57 denn das ist für den Umgang der Fasti mit dem augusteischen Monument letztlich irrelevant. Hier geht es um Monumentalität, nicht um administrative Details (Ov. fast. 5,553-558 = Lugli I68):

Et deus est ingens et opus: debebat in urbe non aliter nati Mars habitare sui.

digna Giganteis haec sunt delubra tropaeis: hinc fera Gradivum bella movere decet, seu quis ab Eoo nos impius orbe lacesset, seu quis ab occiduo sole domandus erit.

Gewaltig ist der Gott und gewaltig sein Tempel. Anders hätte Mars (auch) in der Stadt seines Sohnes nicht wohnen dürfen. Zu diesem Heiligtum gehören Trophäen aus Gigantenkämpfen; Gradivus mag von hier aus wilde Kriege beginnen, sei es, dass ein ruchloser (Feind) uns im Osten reizt, sei es, dass einer im Westen unterworfen werden muss.

5I Der Thesaurus linguae Latinae kennt eine Fülle von Belegen, beginnend mit den Annalen des Ennius: Thes. ling. Lat. 2, p. 592,55-66.

52 Die Junktur bellica signa findet sich in der erhaltenen lateinischen Literatur zuerst hier, später sind damit die Feldzeichen gemeint (z. B. Stat. silv. 3,2,92), so dass eventuell auch schon bei Ovid neben der allgemeinen Beschreibung von Mars' Auftreten ein Hinweis auf die von Parthern zurückgewonnenen Feldzeichen mitschwingt.

53 Klodt (I998), zum Augustusforum: I2-2I (weitgehend paraphrasierend und die Realien erläuternd).

54 Zur Bedeutung der Ultor-Thematik siehe Siebler (I988) I40-I68; außerdem Buchner (I96I).

55 Vgl. Siebler (1988).

56 Thes. ling. Lat. 2, p. 1392,34-43.

57 Dazu ausführlich Spannagel (I999). 
Damit trifft Ovid in poetischer Umschreibung, nicht zuletzt durch die Verwendung des kriegerischen Epithetons Gradivus, ${ }^{8}$ exakt eine der Bestimmungen, die Augustus für die künftige Funktion seines Forums hinaus getroffen hatte (Suet. Aug. 29 = Lugli I65):

Sanxit ergo, ut de bellis triumphisque hic consuleretur senatus, prouincias cum imperio petituri hinc deducerentur, quique uictores redissent, huc insignia triumphorum conferrent.

Er legte also fest, dass hier der Senat über die Kriege und Triumphe beraten sollte, dass von hier die, die sich mit einem Imperium in die Provinzen aufmachten, ausgesandt werden sollten und dass die, die als Sieger heimgekehrt seien, hier ihre Triumphinsignien aufstellen sollten.

Die richtige Umsetzung dieser Programmatik stellt auch Mars fest, der sich über die Details der architektonischen Ausstattung genau informiert, die diese Botschaft eines auf Siegen basierenden Friedens transportiert. Das macht nicht zuletzt die äußerst seltene Apostrophe armipotens 59 deutlich: Nachdem am Anfang akustische Eindrücke im Vordergrund gestanden hatten, geht es nun um die erzählerische Organisation des Blickes. Mars mustert das Ensemble von oben nach unten, von den Victorien am Giebel zu den Beutewaffen in den unteren Regionen (Ov. fast. 5,559-562 = Lugli I68):

Perspicit Armipotens operis Fastigia summi,

et probat invictas summa tenere deas;

perspicit in foribus diversae tela figurae,

armaque terrarum milite victa suo.

Der Waffengott erhebt den Blick zum Giebel seines Tempels, und er ist damit zufrieden, dass die unbesiegten Göttinnen die höchsten Stellen innehaben. An den Türen sieht er Waffen von verschiedener Gestalt und aus (allen) Ländern Rüstungen, die durch seine Soldaten erbeutet wurden.

Sodann fällt der Blick des Gottes auf die doppelte Statuengalerie, die sich über dem Boden des Forums zu beiden Seiten in den Kolonnaden erstreckt, um dann - in einer erneuten Hebung des Blickes - von der ikonographischen Repräsentation samt ihrer epigraphischen Unterstützung zur Inschrift auf dem Tempel zu gelangen (Ov. fast. 5,563-568 = Lugli I68):

Hinc videt Aenean oneratum pondere caro

et tot Iuleae nobilitatis avos;

hinc videt Iliaden umeris ducis arma ferentem, claraque dispositis acta subesse viris.

spectat et Augusto praetextum nomine templum, et visum lecto Caesare maius opus.

58 Serv. Aen. I,292 Mars enim cum saevit Gradivus dicitur, cum tranquillus es Quirinus.

59 Zuvor nur je einmal bei Lukrez und Vergil für Mars belegt: Thes. ling. Lat. 2, p. 6I7, 8-22. 
Hier sieht er Aeneas, mit seiner teuren Last beladen, und so viele Ahnen des julischen Adels; hier sieht er den Sohn der Ilia, wie er die Waffen des (besiegten feindlichen) Führers auf den Schultern trägt und wie die Ruhmestaten der Reihe nach unter jedem Helden (in einer Inschrift aufgezeichnet) sind. Er sieht auch, dass die Front des Tempels mit dem Namen des Augustus versehen ist, und wenn er den Namen des Caesar gelesen hat, scheint ihm das Heiligtum (noch) wertvoller.

Selbst Mars braucht also die Unterstützung durch eine epigraphische Erläuterung, so dass die kommunikative Bedeutung der Weihinschrift als Medium der autoritativen Interpretation (und pars pro toto auch der übrigen Inschriften), die Augustus hatte anbringen lassen, unterstrichen wird. Der tatsächliche Inschriftentext lautet nach der Rekonstruktion durch Géza Alföldy folgendermaßen: ${ }^{\circ 0}$

[Imp(erator) Caesar Divi f(ilius) Augustus pontifex maximus imp(erator) XIII]I c[o(n)s(ul) XIII tribunicia potestate XXI pater patriae]

[ex manibiis] [dono dedit]

[idemque cum C(aio) et L(ucio) Caesaribus filiis co(n)s(ulibus) designatis principibus iuventutis dedicavit]

Anders als Augustus selbst spricht Ovid nur vom Herrscher, nicht von dessen Enkeln. Denn in der Zwischenzeit waren diese beiden Träger der Zukunftshoffnung unerwartet verstorben und Tiberius an ihre Stelle getreten. In der Ars amatoria, noch vor den dynastischen Katastrophen von Limyra und Marseille, hatte Ovid problemlos die enge Verbindung zwischen Augustus, Mars und dem Enkel Gaius Caesar herstellen können (Ov. ars I,I77-204): ${ }^{\text {I }}$

Ecce, parat Caesar domito quod defuit orbi

Addere: nunc, oriens ultime, noster eris.

Parthe, dabis poenas: Crassi gaudete sepulti,

Signaque barbaricas non bene passa manus.

Ultor adest primisque ducem profitetur in annis,

Bellaque non puero tractat agenda puer.

Sieh, Caesar schickt sich an, die Welteroberung zu vervollständigen. Jetzt, fernster Osten, wirst du unser sein. Parther, du wirst bestraft werden. Freut euch, ihr bestatteten Helden des Crassus und ihr Feldzeichen, denen Barbarenhände Schimpf angetan haben! Der Rächer ist da, lässt trotz seiner Jugend schon den Feldherrn erkennen und übernimmt als Knabe die Führung eines Krieges, der nicht Sache eines Knaben ist.

Es scheint, dass Ovid in konkreterer Weise seinen Text an das Augustusforum angeschlossen hat, als bisher in der Forschung bedacht wurde. So verweist ultor adest zum einen auf den Auftrag an Gaius, Rache für Carrhae zu nehmen, zum anderen aber natürlich auf

6o Alföldy (I992) I7-32; vgl. Alfödy (I991) 289-324.

6I Vgl. die ausführliche Erörterung bei Schmitzer (2002). 
Mars Ultor. Denn Augustus hatte den Mars Ultor-Tempel nicht nur eng mit seinen Enkeln ideologisch verknüpft, sondern ihn auch zum Ausgangspunkt für militärische Missionen bestimmt (Cass. Dio 55,I0,2 = Lugli I66): ${ }^{62}$

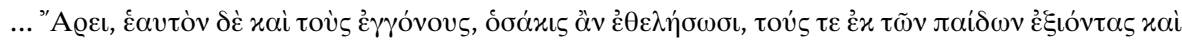

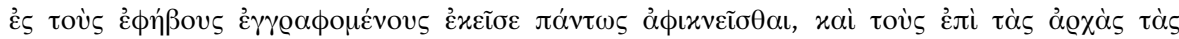

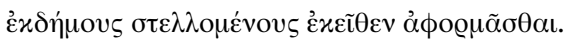

... für Mars und dass er und seine Enkel, sooft sie wollten, dorthin gehen dürften, während diejenigen, die den Stand der Kinder verließen, dort unter die Jungmänner eingeschrieben werden sollten und dass die, die zu auswärtigen Kommanden ausgeschickt wurden, von hier aus aufbrechen sollten.

Der Ultor ist also auch in der Weise zugegen, dass der Rächergott dem Auszug des Rächers der Schmach von Carrhae zusieht und zur Seite steht. Damit wird die räumliche Situation des Augustusforums und ihre politische Zweckbestimmung in den poetischen Text integriert und die unmittelbare visuelle Kommunikation zwischen jugendlichem Feldherrn und dem helfenden Gott durch den Dichter simuliert (Ov. ars I,I83-204):

Parcite natales timidi numerare deorum:

Caesaribus virtus contigit ante diem.

Ingenium caeleste suis velocius annis

Surgit, et ignavae fert male damna morae.

Parvus erat, manibusque duos Tirynthius angues

Pressit, et in cunis iam Iove dignus erat.

Nunc quoque qui puer es, quantus tum, Bacche, fuisti,

Cum timuit thyrsos India victa tuos?

Auspiciis annisque patris, puer, arma movebis,

Et vinces annis auspiciisque patris:

Tale rudimentum tanto sub nomine debes,

Nunc iuvenum princeps, deinde future senum;

Cum tibi sint fratres, fratres ulciscere laesos:

Cumque pater tibi sit, iura tuere patris.

Induit arma tibi genitor patriaeque tuusque:

Hostis ab invito regna parente rapit;

Tu pia tela feres, sceleratas ille sagittas:

Stabit pro signis iusque piumque tuis.

Vincuntur causa Parthi: vincantur et armis;

Eoas Latio dux meus addat opes.

Marsque pater Caesarque pater, date numen eunti:

Nam deus e vobis alter es, alter eris.

62 Zum Bericht des Cassius Dio (55,I0,I-9) siehe Swan (2004) 93-IOI. 
Zählt nicht ängstlich die Geburtstage von Göttern! Caesaren wird Tüchtigkeit vor der Zeit zuteil. Der vom Himmel stammende Geist erhebt sich schneller, als die gesetzlichen Jahre kommen, und erträgt schlecht den Verlust, den träges Warten bedeutet. Klein war Hercules, und erdrückte doch mit den Händen zwei Schlangen; so war er schon in der Wiege seines Vaters Juppiter würdig. Und du, Bacchus, noch heute ein Knabe, wie klein warst du erst, als das besiegte Indien vor deinen Thyrsusstäben zitterte? Vom Glück gesegnet und jung, wie dein Vater war, wirst du, Knabe, den Krieg beginnen und siegen, jung und vom Glück gesegnet wie er. Solch einen Anfang bist du uns als Träger eines so großen Namens schuldig, jetzt der erste unter den Jünglingen, später der erste im Rat der Alten! Da du Brüder hast, räche die Kränkung der Brüder; da du einen Vater hast, schütze die Rechte des Vaters! Die Waffen hat dir der Vater des Vaterlandes, der zugleich der deine ist, angelegt. Der Feind raubt das Königtum gegen den Willen deines Vaters! Du wirst heilige Waffen tragen, er fluchbeladene Pfeile; vor deinen Feldzeichen werden Recht und frommer Sinn stehen. Das Recht entscheidet gegen die Parther, mögen nun auch die Waffen gegen sie entscheiden! Möge mein Feldherr Macht und Reichtum des Orients Latium einverleiben! Vater Mars und Vater Caesar, schenkt ihm bei seinem Aufbruch euren göttlichen Beistand, denn einer von euch ist schon ein Gott, der andere wird es werden.

In dieser Passage ist implizit die Aufbewahrung der Feldzeichen von Carrhae im Mars U1tor-Tempel vorausgesetzt. Der Mars pater, die Inkarnation iulischer Familiengenealogie und der Ikonographie seines Kultbildes, soll für einen erneuten Parthererfolg bürgen. Damit ist im ideologischen und archiktektonischen Rahmen des Augustusforums der Blick der Ars amatoria zukunftsorientiert.

In den Fasti dagegen setzt Ovid aus genosspezifischen Gründen - in einer aus der jüngeren Vergangenheit abgeleiteten Aitiologie -, aber auch wegen der veränderten Rahmenbedingungen andere Akzente. War in der Ars die von Augustus veranlasste Inschrift narrativ umgesetzt und fortgeführt worden, so ist sie in den Fasti explizit, wenn auch nur selektiv genannt und wird im Fortgang aitiologisch erläutert. Folgt man der fiktiven Datierung des Textes, die durch das unmittelbar vor der Schlacht von Philippi geäußerte wörtliche Zitat, das Augustus in den Mund gelegt ist, bestimmt wird, dann ist das der früheste Beleg für die Epiklese des Mars als Ultor (Ov. fast. 5,569-578 = Lugli ı68):

Voverat hoc iuvenis tum cum pia sustulit arma:

a tantis princeps incipiendus erat.

ille manus tendens, hinc stanti milite iusto,

hinc coniuratis, talia dicta dedit:

„si mihi bellandi pater est Vestaeque sacerdos

auctor, et ulcisci numen utrumque paro,

Mars, ades et satia scelerato sanguine ferrum, stetque favor causa pro meliore tuus.

templa feres et, me victore, vocaberis Ultor.“

voverat, et fuso laetus ab hoste redit. 
(Augustus) hatte es als junger Mann gelobt, als er aus Pietät zu den Waffen griff. Mit einem solchen Werk musste der Princeps (seinen Weg) beginnen. Hier standen die Soldaten der gerechten Sache, dort die Verschworenen, (da) erhob er seine Hände und rief: „Wenn (Caesar als) mein Vater und (als) Priester der Vesta mich zum Kampfe ruft und ich mich aufmache, beide Gottheiten zu rächen, Mars, steh mir bei und sättige das Eisen mit Verbrecherblut. Deine Gnade stehe auf der Seite der besseren Sache! Einen Tempel wirst du erhalten, und wenn ich siege, wirst du den Beinamen ,Rächer' erhalten." Das war sein Gelübde, und nach seinem Siege über den Feind kehrte er im Jubel heim.

Durch die rahmende Wiederaufnahme von voverat ist dieser Teil als abgeschlossen markiert. Ovid hat die offizielle Begründung paraphrasierend wiedergegeben oder damit einen über den Parthersieg - und dessen Repräsentation durch die Feldzeichen im Zentrum der gesamten Anlage - eher in den Hintergrund getretenen Aspekt wieder Prominenz verliehen, so dass das ursprüngliche Aition (das allerdings innenpolitisch durchaus heikel war: ein Siegesmonument aus den Bürgerkriegen gab es ja sonst nicht in solch expliziter Form) ${ }^{6_{3}}$ gegenüber dem sekundären wieder in Erinnerung gerufen wurde. Dann fährt er ex persona poetae fort und liefert in gut hellenistischer Tradition den zweiten Teil der Doppelerklärung (Ov. fast. 5,579-596):

Nec satis est meruisse semel cognomina Marti: persequitur Parthi signa retenta manu. gens fuit et campis et equis et tuta sagittis et circumfusis invia fluminibus; addiderant animos Crassorum funera genti, cum periit miles signaque duxque simul. signa, decus belli, Parthus Romana tenebat, Romanaeque aquilae signifer hostis erat; isque pudor mansisset adhuc, nisi fortibus armis Caesaris Ausoniae protegerentur opes.

ille notas veteres et longi dedecus aevi sustulit: agnorunt signa recepta suos. quid tibi nunc solitae mitti post terga sagittae, quid loca, quid rapidi profuit usus equi?

Parthe, refers aquilas, victos quoque porrigis arcus: pignora iam nostri nulla pudoris habes.

Es genügte aber nicht, für Mars den Beinamen einmal verdient zu haben; er ging aus auf die Feldzeichen, die von der Hand der Parther zurückgehalten wurden. Diese waren ein Volk, das sicher war durch seine (weiten) Ebenen, seine Pferde und seine Pfeile, und das unerreichbar war durch die Flüsse, die es umgeben. Der Tod des Crassus und seines Sohnes hatte dem Volke den Mut gestärkt, als zu gleicher Zeit die Truppen, der Feldherr und

63 Vgl. Zanker (1987) 90-96; 196-204. 
die Zeichen untergingen. Römische Feldzeichen, die Sinnbilder der Waffenehre, besaß der Parther, und ein Feind war der Feldzeichenträger des römischen Legionsadlers! Und diese Schmach hätte bis heute angedauert, wenn das Reich des Abendlandes nicht von Caesars tapferen Waffen geschützt würde. Er hat die alte Schande und die Schmach eines (ganzen) langen Zeitalters getilgt, die zurückgewonnenen Feldzeichen erkannten ihre (alten Besitzer) wieder. Was haben dir, Parther, die Pfeile nun genützt, die ihr gewöhnlich von hinten (auf den Gegner) schießt, was das Gelände, was der Gebrauch der schnellen Pferde? Du gibst die Adler wieder, und du reichst auch die besiegten Bogen her. Jetzt besitzest du keine Zeichen unserer Schande mehr!

Das ist sicher alles unbestreitbar richtig und Augustus hätte kaum tatsächliche Einwände gegen Ovids Darstellung erheben können. Dennoch geht mit der Vertextlichung auch eine Umakzentuierung einher: Augustus hatte - entsprechend seiner auch sonst verfolgten Maxime - den konkreten historischen Bezug, insbesondere auf die Siege im Bürgerkrieg, zurückgenommen und die tatsächlichen Ereignisse mythisch überhöht. Ovid lenkt den Blick auf die beiden zeitgeschichtlichen Ereignisse zurück, überhöht sie aber in anderer Weise, indem er - in für die Fasti singulärer Form - den zuständigen Gott selbst zum Publikum werden lässt und komplementär dazu den Princeps zum eigentlichen Träger der Handlung.

Wie heikel eine solche ausdrückliche Erwähnung der Bürgerkriegszeit war, zeigt ein Zeugnis aus der Zeit des Tiberius. Als nach dem Prozess gegen Piso und dessen Tod der Senat Ehrungen für das Kaiserhaus beschließen wollte, schritt der princeps ein (Tac. ann. 3,I8 = Lugli I 85$):^{64}$

Atque idem, cum Valerius Messalinus signum aureum, in aede Martis Ultoris, Caecina Severus aram Ultionis statuendam censuissent, (Tiberius) prohibuit, ob externas ea victorias sacrari dictitans, domestica mala tristitia operienda.

Und als Valerius Messalinus ein goldenes Standbild im Tempel des Mars Ultor errichten lassen wollte, Caecina Severus einen Altar der Rache, verbot das Tiberius, da er betonte, dieser Tempel sei wegen der auswärtigen Siege geweiht, die häuslichen Übel müssen mit Trauer bedeckt werden.

Ovid legt mit seiner prononcierten, die zeitliche in eine wertende Abfolge umgestaltenden Darstellung also auch den Finger auf die düsteren Umstände der Weihung und Entstehung, die nicht mehr in jeder Hinsicht opportun erschienen. Der Dichter schließt mit der nochmaligen Erwähnung des doppelten Aitions und der ludi, die sich um die Tempelweihe rankten (Ov. fast. 5,595-598):

64 Woodman u. Martin (I996) ad loc. 
Rite deo templumque datum nomenque bis ulto,

et meritus voti debita solvit honor.

sollemnes ludos Circo celebrate, Quirites:

non visa est fortem scaena decere deum.

Mit Recht wurde der Tempel und der Name dem Gotte gegeben, der (uns) zweimal rächte.

Die Verpflichtung des Gelübdes wurde durch verdiente Ehrung getilgt. Begeht im Circus

die feierlichen Spiele, Quiriten: die Bühne schien nicht zu dem Kriegsgott zu passen.

Auch wenn Mars im Laufe der Schilderung als Handelnder aus dem Text verschwunden ist und nur noch als Empfänger von Augustus' Gelübde fungiert, kann nach der Logik der Darstellung kein Zweifel daran bestehen, dass Intention und Ausführung die Billigung des Gottes finden, die ultio Augusta als Voraussetzung für die pax Augusta.

Mars ist vielleicht nicht der erste Rezipient, aber auf jeden Fall der höchstrangige und wichtigste. Ovid präsentiert uns ein monumentales, menschenleeres, ganz von der ultio-Thematik geprägtes Augustusforum, gewissermaßen die Perspektive der archäologischen Modelle (wie desjenigen in der Ausstellung der Fori Imperiali). ${ }^{65}$ In der Realität kann das allenfalls an einem frühen Frühlings- oder Sommermorgen so gewesen sein, bevor all diejenigen sich eingefunden hatten, die in den Gedichten Martials, den Angaben der pompeianischen und herculanischen Tabulae oder den en passant sich ergebenden Eindrücken bei Sueton, Tacitus und Cassius Dio bezeichneten Personengruppen das Augustusforum bevölkerten.

Ovid kommt nach der Ars amatoria und den Fasti noch ein drittes Mal auf das Augustusforum zu sprechen, nämlich in der großen Exilelegie trist. 2, seiner an Augustus gerichteten Rechtfertigung insbesondere gegen auf die Ars amatoria zielende moralische Vorwürfe. Auch die Tempel sind in die Verteidigungsstrategie einbezogen (trist. 2,287): Quis locus est templis augustior? Und dennoch sind diese erhabenen, augustusgemäßen Stätten voll von erotischen Verlockungen, wie nicht zuletzt das ikonographische Programm des Augustusforums zeigt (Ov. trist. 2,295f. = Lugli I76):66

Venerit in magni templum, tua munera, Martis, stat Venus Vltori iuncta, uir ante fores.

Mag der Besucher in den Tempel des großen Mars kommen, deine Gabe, dann steht Venus verbunden mit dem Ultor da, ihr Mann aber draußen vor der Tür.

Dass der durch das homerische Gelächter berühmt gewordene Ehebruch von Ares und Aphrodite und der Betrug an Hephaistos nun aus der Götterburleske in die Apologie überführt wird und aus dem staatstragenden Monument ein Ausgangspunkt für unmoralische Gedanken wird, kann kaum im Sinne des Augustus gewesen sein. Ovid gelangt dahin,

65 Ungaro (2007).

66 Ingleheart (2010) 251; 254-256. 
indem er die von Varro in seiner Unterscheidung von poetischer und politischer Religion gesetzten Grenzen ignoriert. ${ }^{67}$

Ovid greift damit einen Gedankengang auf, der schon in der Ars amatoria angeklungen war, in einer Passage unmittelbar vor dem bereits besprochenen Propemptikon für Gaius Caesar (Ov. ars I,79-88):

Et fora conveniunt (quis credere possit?) amori:

Flammaque in arguto saepe reperta foro:

Subdita qua Veneris facto de marmore templo

Appias expressis aera pulsat aquis,

Illo saepe loco capitur consultus Amori,

Quique aliis cavit, non cavet ipse sibi:

Illo saepe loco desunt sua verba diserto,

Resque novae veniunt, causaque agenda sua est.

Hunc Venus e templis, quae sunt confinia, ridet:

Qui modo patronus, nunc cupit esse cliens.

Und auch die Foren passen für die Liebe - wer wollte es glauben? Und die Liebesglut wird häufig auf dem spitzzüngigen Forum gefunden. Wo unterhalb des aus Marmor errichteten Tempels der Venus die Appias ihre Wasser in Stößen in die Luft schickt, an jenem Ort wird häufig der Rechtskundige von Amor ergriffen. Und wer für andere Vorsorge leistet, leistet sie nicht für sich selbst. An jenem Ort fehlen häufig dem Beredten die Worte, neue Sachverhalte stellen sich ein, man muß in eigener Sache tätig werden. Diesen verlacht Venus aus den Tempeln, die angrenzen. Wer eben noch Patron war, möchte nun Klient sein.

Als Ovid diese Verse schrieb, war Augustus wohl gerade mit der Vorbereitung der Einweihungsfeiern befasst, das Forum selbst war ja schon in Benutzung. Das Forum Iulium und das Forum Augustum - die fora des Textes, die einander benachbart waren (confinia) - waren gemeinsam den beiden göttlichen Stammeltern Mars und Venus geweiht. Während aber Mars im Tempel der Venus Genetrix nichts zu suchen hatte, war beim Mars UltorTempel auch Venus zugegen. Damit bekommen die Plurale fora und templis Signifikanz als Verweise auf die beiden neuen, von Augustus vollendeten öffentlichen Repräsentationsräume. Was hindert also daran anzunehmen, dass Ovid hier einen kecken literarischen Usurpationsversuch vornimmt? ${ }^{68}$

*

67 Deutlich affirmativer ist Zanker (I968) I9, der v.a. auf den Concordia-Aspekt abhebt; vgl. Siebler (I988) I30-I36.

68 Von einer strengen Scheidung auch der Eindrücke, die von den beiden Fora ausgingen, spricht zuletzt noch Geiger (2008) I8If. 
Die monumentale Architektur ist damit wieder in die Alltagswelt, die des Flirts und der amourösen Abenteuer, zurückgeholt. Zugleich zeigt sich an den unterschiedlichen Perspektiven, die sich allein schon aus Ovids Dichtungen auf das Augustusforum ergeben, dass diese Texte alles andere als Architekturbeschreibungen sind, sich also zur archäologischen Rekonstruktion ${ }^{6} 9$ nur in höchst begrenztem, interpretatorisches Fingerspitzengefühl erforderndem Umfang eignen. Diese poetischen Texte verfolgen ein eigenes Konzept, das nicht auf enzyklopädische Totalität abzielt, sondern sich aus den jeweiligen kommunikativen Zusammenhängen ergibt. Darin aber nähern sie sich auch den tatsächlichen Wahrnehmungs- und Aneignungsweisen an, mit denen sich die Römer des Areals bedienten. Und nur selten kam es zu einer Überschneidung der Sphären wie wir sie in der eingangs behandelten Anekdote von Claudius als Übergang vom Ernsthaften zum Lächerlichen kennen gelernt haben.

Doch dieser Claudius erlebte auf dem Forum seines Großonkels nicht nur glückliche Momente (Suet. Claud. I3,I-2):

Nec tamen expers insidiarum usque quaque permansit, sed et a singulis et per factionem et denique ciuili bello infestatus est. e plebe homo nocte media iuxta cubiculum eius cum pugione deprehensus est; reperti et equestris ordinis duo in publico cum dolone ac uenatorio cultro praestolantes, alter ut egressum theatro, alter ut sacrificantem apud Martis aedem adoreretur.

Und auch von Nachstellungen blieb er nicht verschont, sondern er wurde von einzelnen, von Gruppen und schließlich in bürgerkriegsartigem Aufruhr bedroht. Ein Mann aus der Plebs wurde mitten in der Nacht neben seinem Schlafzimmer mit einem Dolch gefasst, man fand zwei aus dem Ritterstand, die in der Öffentlichkeit mit einem Dolch und Jagdmesser auf ihn wie zur Begrüßung warteten, der eine, um ihn beim Verlassen des Theaters anzugreifen, der andere beim Opfer beim Marstempel.

Es ist eine treppenwitzartige Wendung, dass just dieser Claudius uns eine bildliche Überlieferung vom Augustusforum und Marstempel als Hintergrund einer Opferszene gegeben hat ${ }^{\circ}$ - und ein solches Opfer hätte ihn beinahe das Leben gekostet, ihn, den Kaiser auf dem Forum eines anderen Kaisers.

69 Vgl. etwa die Verwendung der Fasti bei Zanker (I988) II9.

70 Zanker (1987) III, Abb. 86: Opferszene vor dem Mars Ultor-Tempel auf einem Relief aus claudischer Zeit. 


\section{Literatur}

Alföldy (I99I)

Géza Alföldy, „Augustus und die Inschriften. Tradition und Innovation“, Gymnasium 98, 289-324.

Alföldy (1992)

Géza Alföldy, Studi sull'epigrafia augustea e tiberiana di Roma, Roma.

\section{Barchiesi (2002)}

Alessandro Barchiesi, „Martial Arts. Mars Ultor in the Forum Augustum: A Verbal Monument with a Vengeance“, in: Geraldine Herbert-Brown (Hg.), Ovid's Fasti. Historical Readings at its Bimillennium, Oxford, I-22.

\section{Bastien (2007)}

Jean-Luc Bastien, Le triomphe romain et son utilisation politique à Rome aux trois derniers siècles de la république, Rome (Collection de L'École Française de Rome 392).

\section{Bömer (1957/58)}

P. Ovidius Naso, Die Fasten. Kommentar von Franz Bömer, Heidelberg.

\section{Buchner (196I)}

Edmund Buchner, Art. Ultor, RE 9A,I, 572-578.

\section{Camodeca (1999)}

G. Camodeca, Tabulae Pompeianae Sulpiciorum. Edizione critica dell'Archivio puteolano dei Sulpicii, Roma.

\section{Carey (2003)}

Sorcha Carey, Pliny's Catalogue of Culture. Art and Empire in the Natural History, Oxford.

\section{Carnabuci (1996)}

Elisabetta Carnabuci, I luoghi dell'amministrazione della giustizia nel Foro di Augusto, Napoli.

\section{Cecamore (2002)}

Claudia Cecamore, Palatium. Topografia storica del Palatino tra III sec. A.C. e I sec. D.C., Roma (Bullettino della Commissione Archeologica Comunale di Roma, Suppl. 9).

\section{Degrassi (1937)}

A. Degrassi, Elogia Fori Augusti, Inscriptiones Italiae: I3, Fasti et Elogia, Roma, I-36.

\section{Edelmann (2003)}

Babett Edelmann, „Arvalbrüder und Kaiserkult“, in: Hubert Cancik, Konrad Hitzl (Hgg.): Die Praxis der Herrscherverehrung in Rom und seinen Provinzen, Tübingen, 189-205.

\section{Frisch (1980)}

Peter Frisch, „Zu den Elogien des Augustusforums“, Zeitschrift für Papyrologie und Epigraphik 39, 9I-98. 


\section{Galinsky (1996)}

Karl Galinsky, Augustan Culture. An Interpretive Introduction, Princeton.

\section{Geiger (2008)}

Joseph Geiger, The First Hall of Fame. A Study of the Statues in the Forum Augustum, Leiden, Boston, Tokyo (Mnemosyne Suppl. 295).

\section{Herbert-Brown (1994)}

Geraldine Herbert-Brown, Ovid and the Fasti. An historical study, Oxford.

\section{Ingleheart (2010)}

Jennifer Ingleheart, A Commentary on Ovid, Tristia, Book 2, Oxford.

\section{Keulen (2009)}

Wytse Keulen, Gellius the Satirist. Roman Cultural Authority in Attic Nights, Leiden (Mnemosyne Suppl. 297).

\section{Kienast (2009)}

Dietmar Kienast; Augustus. Prinzeps und Monarch, 4. bibliographisch aktualisierte und um ein Vorwort ergänzte Auflage, Darmstadt.

\section{Kierdorf (1992)}

Sueton, Leben des Claudius und Nero. Textausgabe mit Einleitung, kritischem Apparat und Kommentar herausgegeben von Wilhelm Kierdorf, Paderborn et al.

\section{Klodt (I998)}

Claudia Klodt, „Platzanlagen der Kaiser in der Beschreibung der Dichter“, Gymnasium I05, $\mathrm{I}-38$.

\section{Koestermann (1963)}

Tacitus, Annalen, hg. von Erich Koestermann, Bd. I, Heidelberg.

\section{Kühnen (2008)}

Angela Kühnen, Die imitatio Alexandri in der römischen Politik (1. Jh. v. Chr.-3. Jh. n. Chr.), Münster.

\section{Larmour (2007)}

David H.J. Larmour, „Holes in the Body. Sites of Abjection in Juvenal's Rome“, in: David H.J. Larmour u. Diana Spencer (Hgg.), The Sites of Rome. Time, Space, Memory, Oxford, I68-2io.

\section{Lhommé (20II)}

Marie-Karine Lhommé, „De l'encyclopédie au glossaire: Festus et son adaption par Paul Diacre“, in: Rolando Ferri (Hg.), The Latin of Roman Lexicography, Pisa, Roma, 29-47.

\section{Linderski (200I)}

Jerzy Linderski, Art. Salii, Der Neue Pauly ıo, I249-I25I. 


\section{Lugli (1965)}

Iosephus Lugli, Fontes ad topographiam veteris urbis Romae pertinentes, Vol. VI,I, liber XVI: Fora imperatorum (Regio VIII), Romae.

\section{Meneghini u. Valenzani (2007)}

Roberto Meneghini u. Riccardo Valenzani, I Fori Imperiali. Gli scavi del Comune di Roma (1991-2007), Roma.

\section{Norden (1939)}

Eduard Norden, Aus altrömischen Priesterbüchern, Lund.

\section{Rich (1998)}

J.W. Rich, Augustus's Parthian Honours, Papers of the British School at Rome 66, 7I-I28.

\section{Riedl (1989)}

Rita Riedl, Mars Ultor in Ovids Fasten, Amsterdam (Heuremata Io).

\section{Scheid (1998)}

John Scheid, Commentarii fratrum Arvalium qui supersunt. Les copies épigraphiques des protocoles annuels de la confrérie arvale (21 av.-304 ap. J.-C.), Rome.

\section{Scheid (2007)}

Res gestae divi Augusti. Hauts Faits du Divin Auguste, texte établi et traduit par John Scheid, Paris.

\section{Schmitzer (2002)}

Ulrich Schmitzer, „Die Macht über die Imagination. Literatur und Politik unter den Bedingungen des frühen Prinzipats“, RhM I45, 28I-304.

\section{Schmitzer (2003)}

Ulrich Schmitzer, „Dichtung und Propaganda im I. Jahrhundert n. Chr.“, in: M. Zimmermann u. G. Weber (Hgg.), Selbstdarstellung, Propaganda, Repräsentation im römischen Kaiserreich des 1. Jhs. n. Chr., Stuttgart (Historia Einzelschriften I64), 205-226.

\section{Schmitzer (2004)}

Ulrich Schmitzer, Friede auf Erden? Latinistische Untersuchungen zur pax Augusta in interdisziplinärer Perspektive, Berlin.

\section{Schmitzer (2007)}

Ulrich Schmitzer, „Ovids Carmentalia - oder: Kann man einem Dichter vertrauen?“, in: Markus Janka, Ulrich Schmitzer u. Helmut Seng (Hgg.), Ovid. Kultur - Werk - Wirkung, Darmstadt, II3-I44.

\section{Schöffel (2002)}

Christian Schöffel, Martial, Buch 8. Einleitung, Text, Übersetzung, Kommentar, Stuttgart (Palingenesia 77).

\section{Siebler (1988)}

Michael Siebler, Studien zum augusteischen Mars Ultor, München I988. 


\section{Stevenson (2004)}

Andrew J. Stevenson, "Gellius and the Roman Antiquarian Tradition“, in: Leofranc Holford Strevens u. Amiel Vardi (Hgg.), The Worlds of Aulus Gellius, Oxford, II8-I55.

\section{Swan (2004)}

Peter Michael Swan, The Augustan Succession. An Historical Commentary on Cassius Dio's Roman History Books 55-56 (9 B.C. - A.D. 14), Oxford.

\section{Spannagel (1999)}

Martin Spannagel, Exemplaria Principis. Untersuchungen zu Entstehung und Ausstattung des Augustusforums, Heidelberg (Archäologie und Geschichte 9).

\section{Tisé (2002)}

Bernadette Tisé, Imperialismo romano e imitatio Alexandri. Due Studi di storia politica, Galatina.

\section{Ungaro (2007)}

Lucrezia Ungaro (Hg.), Il Museo dei Fori Imperiali nei Mercati di Traiano, Milano.

van Heck (2002)

Adrianus van Heck, Breviarium urbis Romae, viatorum in usum composuit A. v. H., 2. Auflage, Leiden, Rom.

\section{Woodman u. Martin (1996)}

A.J. Woodman u. R.H. Martin, The Annals of Tacitus, Book 3, Cambridge.

\section{Wolf (2010)}

Joseph Georg Wolf (Hg.), Neue Rechtsurkunden aus Pompeji. Tabulae Pompeianae Novae. Lateinisch und Deutsch, Darmstadt.

\section{Zanker (I968)}

Paul Zanker, Forum Augustum. Das Bildprogramm, Tübingen (Monumenta Artis Antiquae 2).

\section{Zanker (1987)}

Paul Zanker, Augustus und die Macht der Bilder, München. 